Research Article

\title{
Theoretical Study of Convection Heat Transfer and Fluid Dynamics in Microchannels with Arrayed Microgrooves
}

\author{
Hai-Ping Hu (D) \\ Department of Marine Engineering, National Taiwan Ocean University, Keelung 20224, Taiwan \\ Correspondence should be addressed to Hai-Ping Hu; hphu@ntou.edu.tw
}

Received 5 July 2020; Revised 18 January 2021; Accepted 22 February 2021; Published 3 March 2021

Academic Editor: Jan Vorel

Copyright (c) 2021 Hai-Ping Hu. This is an open access article distributed under the Creative Commons Attribution License, which permits unrestricted use, distribution, and reproduction in any medium, provided the original work is properly cited.

\begin{abstract}
The analysis of heat transfer and fluid dynamics in a microchannel is currently one of numerous important research areas. The present study investigated the heat transfer and fluid dynamics in a microchannel with arrayed microgrooves. The boundary conditions in the microchannel and microgrooves were the velocity slip condition and temperature jump condition. The numerical calculations in the present study were based on a new numerical analysis scheme that was used to name modified pointmatching methods. The methods are applied to calculate the two items coefficients of the series expressions of velocity in the microchannel with microgrooves. The methods analyzed four items coefficients of the series expressions of temperature in the microchannel with microgrooves. In addition, the fluid velocity, temperature distribution, Knudsen number effect, fluid friction factor in the microchannel, and heat transfer were discussed.
\end{abstract}

\section{Introduction}

The significance of high heat fluxes in micro-electromechanical system (MEMS), microdevice fabrication, bioengineering, and other devices has caused microscale heat transfer to be the subject of numerous recent studies. Research on microchannel flow behavior in microregions has been fundamental for developing this important knowledge and is of value in heat transfer engineering. Microfluid systems typically have characteristic lengths in the order of $1-100 \mu \mathrm{m}$. The Knudsen number $(K n)$, which characterizes the effect of rarefaction, is defined as the ratio of the molecular mean free path in the order of $100 \mathrm{~nm}$. Rarefied gaseous flows in these microgeometries have frequently been observed, i.e., for a microchannel system, the Knudsen number, which is under the range from $10^{-3} \leq K n \leq 10^{-1}$ belonging to the velocity-slip and temperature-jump regime. Consequently, a microchannel system should further consider the effects of the slip flow and temperature jump of the surface. The pioneer research and analyses of the laminar heat transfer in a slip-flow regime were performed by Sparrow and Lin [1] and Inman [2] for tubes with constant heat flux and a parallel plate channel or a circular tube with isothermal conditions.
Next, Larrode et al. [3] considered the temperature jump condition and found that the effect of the fluid-wall interaction is also important. Natural convection of an enclosed fluid has received considerable attention in recent years due to its wide application in engineering problems. Earlier work on the isothermal parallel-plate channels was reported by Elenbaas [4]. Further investigations have been carried out for different thermal boundary conditions. An excellent review is given in the literature by Gebhart et al. [5]. All of the previous investigations were conducted at the macroscale; therefore, natural convection flow in microscale systems should be studied extensively. Aung [6] describes the influences of rarefaction and fluid-wall interaction on steady and fully-developed natural convection in microchannels. The results of Aung's study can be applied to microheat exchangers.

Furthermore, $\mathrm{Yu}$ and Ameel [7] studied slip flow heat transfer in microchannels and found that the heat transfer increases, decreases, or remains unchanged when compared to nonslip flow conditions. Kavehpour et al. [8] investigated the effects of compressibility and rarefaction in microchannel heat transfer under slip flow and discussed the compressibility and rarefaction under different Reynolds 
numbers. Vasudeviah and Balamurugan [9] investigated the convective heat transfer in a microchannel with respect to rarefied gas flows and obtained analytical solutions for the mean Nusselt number. Tunc and Bayazitoglu [10] researched the microchannel heat transfer under the slip flow and constant heat flux problem and stated that increasing the rarefaction has a decreasing effect on the heat transfer. Ghodoossi and Egrican [11] studied the heat transfer in a rectangular microchannel for a slip flow regime. In the present paper, the integral transform method was applied to derive the velocity and temperature distributions and find the average Nusselt number. Zhu and Liao [12] studied the laminar forced convective heat transfer of a gas flowing through a microchannel with an axially-constant heat flux and circumferentially-varied wall temperature boundary condition in the slip-flow and the temperature-jump regime. Bahrami et al. [13] investigated the fully-developed laminar flow in smooth channels of arbitrary cross-sections of microchannel using existing analytical solutions for fluid flow to derive the $f$ Re values. Zhang et al. [14] researched the experimental study on liquid flow and heat transfer in rough microchannels. The results indicate that the surface roughness plays a significant role in the process of laminar flow and heat transfer in microchannels. In microchannels with roughness characteristics, the Poiseuille number of liquid laminar flow relies not only on the cross section shape of the rough microchannels but also on the Reynolds number of liquid flow. Elguennouni et al. [15] investigated the thermal lattice Boltzmann model for nonisothermal gas flow in a two-dimensional microchannel. The thermal Lattice Boltzmann method is used for the simulation of a gas microflow. A two-dimensional heated microchannel flow driven by a constant inlet velocity profile and nonisothermal walls is investigated numerically.

Important numerical and experimental studies with hydrothermal analyses under different boundary conditions and various high-performance obstacles are addressed by Peiravi and Alinejad [16]. Alinejad and Peiravi [17] investigated three-dimensional numerical simulation of a Newtonian droplet impact and break on two square cylinders based on dynamic contact angle of droplet at the spatial interface between two solid-fluid phases. Asshaari et al. [18] investigated nanofluids containing carbon nanotubes with radiation and velocity slip effects, in case of a moving plate containing both single-walled and multiwalled carbon nanotubes, in the presence of thermal radiation and the slip effect. In the recent research, Yuan et al. [19] created the computational simulation of MHD nanofluid which flows past a baffled U-shaped enclosure by using Koo-Kleinstreuer-Li (KKL) method. The U-shaped enclosure, nanofluid, magnetic field, and baffles are all included in the investigation. Mohebbi and Rashidi [20] investigated natural convection of a nanofluid inside of an L-shaped enclosure. The results of the study presented that fluid flow field and convection heat transfer were significantly influenced by the heat source position. For all aspect ratios, when a heat source is positioned in the lower and beside the left wall inside the cavity, the Nusselt number is obtained.
Although numerous researches about microchannel or nanofluids under different conditions have been discussed, the microchannel heat and flow of arrayed microgrooves with different aspect ratios and cross-sectional areas are also worth discussing, particularly in thermal and flow analysis. The present paper thus investigated the heat and fluid flow of a microchannel with microgrooves, i.e., a microchannel with microgrooves positioned along the $X$-direction with a flow moving in the $Z$-direction. The characteristics of the heat transfer and fluid dynamics in the microchannel, e.g., the Knudson number and the boundary conditions of velocity slip and temperature jump, were considered in the present research.

\section{Description of the Physical Model}

The present study considered a steady-state fully developed laminar fluid flow in a two-dimensional microchannel. The height of the channel was $a H$, and it was assumed that the left side and the right side of the microchannel were infinite. The microchannel included parallel grooves arrayed along the $X$ direction. The height of the grooves was $b H$, and the width of the grooves was $2(1-c) H$. The cross section of the microchannel and the physical model are shown in Figure 1. The direction of the flow was normal in this paper ( $Z$-dimensional). Since the velocity of a fluid is very slow, it was assumed that the inertia force was negligible. The flow is hydraulically and thermal fully developed laminar, and it is incompressible and has constant properties. For such a flow, the pressure gradient along the $Z$-dimension is a constant. The momentum equation for the velocity of the microfluid could be expressed as

$$
\frac{\partial^{2} W}{\partial X^{2}}+\frac{\partial^{2} W}{\partial Y^{2}}=-\frac{1}{\mu} \frac{\mathrm{d} P}{\mathrm{~d} Z}
$$

Energy equation:

$$
\alpha\left(\frac{\partial^{2} T}{\partial X^{2}}+\frac{\partial^{2} T}{\partial Y^{2}}\right)=W(X, Y) \frac{\mathrm{d} T}{\mathrm{~d} Z}
$$

where $X$ is the horizontal coordinate axis; $Y$ is the vertical coordinate axis; $W$ is the $2 \mathrm{D}$ velocity distribution in the $Z$ direction; $W(X, Y) ; Z$ is the direction normal to the paper surface; and $P$ is the pressure.

The Knudsen number $(K n)$ is a dimensionless parameter used in discussions on the rarefaction effect in a microchannel. This physical parameter is defined as $K n \equiv l L$, where $l$ is the molecular mean free path, defined as the average distance moved in two collisions of gas molecules, and $L$ is the characteristic length of the microchannel. The stereogram shown in Figure 1 is represented as a 2D diagram.

Considering the factor of geometric symmetry, this study focused on the L-shaped region, represented as slashes in Figure 2. This L-shaped region $\left(W_{z}(X, Y)\right)$ was decomposed into two connected rectangular regions using the domain decomposition method, and the two regions were computed simultaneously. The dimensionless parameters were imported, in which $w=-W /\left(H^{2} \mathrm{~d} p / \mu \mathrm{d} Z\right)$ and $\theta(x, y)=(T(x, y)$ 


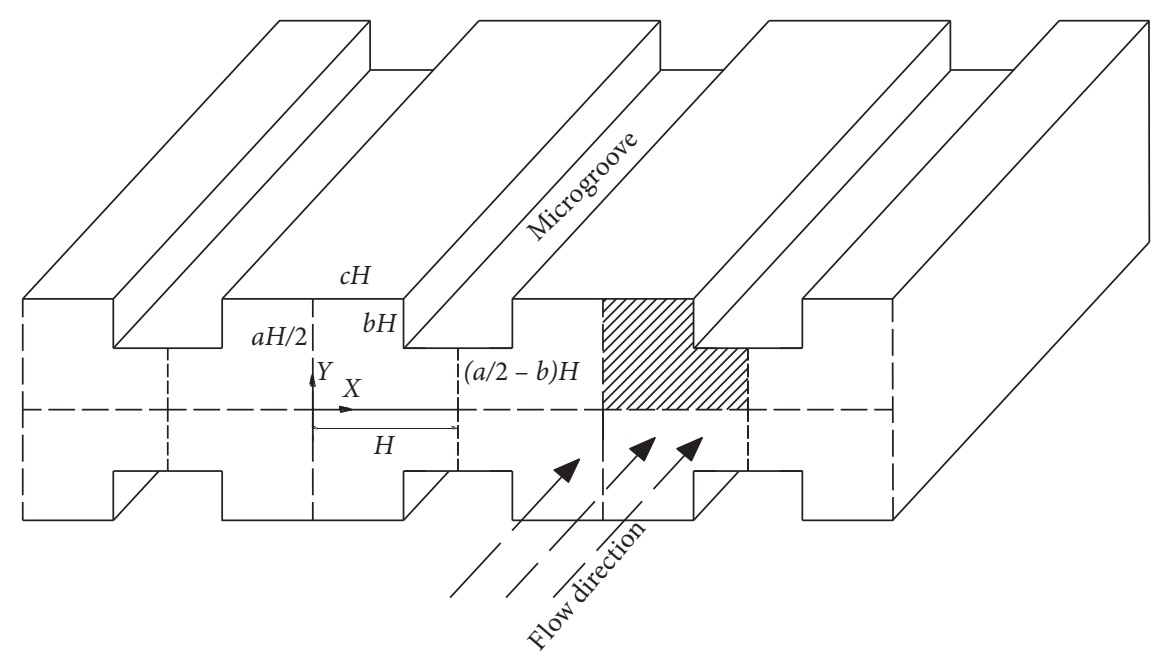

FIGURE 1: 3D schematic diagram of a microchannel with arrayed microgrooves.

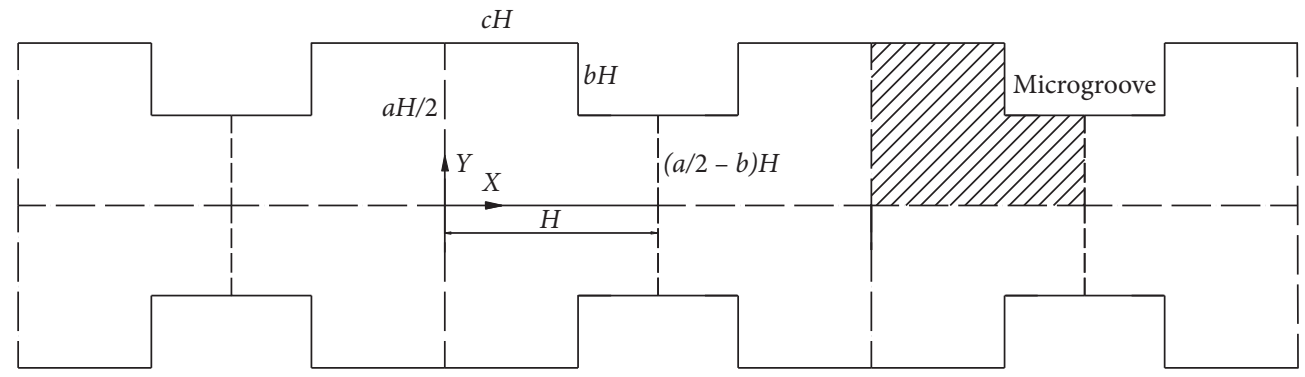

FIGURE 2: Diagrammatic sectional drawing of a microchannel with arrayed microgrooves.

$\left.-T_{\text {wall }} / H q_{m} L / k \bar{w}\right)$. The L-shaped region was decomposed, and the noncoherent boundary conditions of the dimensionless slip velocity and the dimensionless temperature after dimensionless parameter displacement are as shown in the following diagram (Figure 3), in which $H$ is the width, $\mu$ is the viscosity coefficient, $q_{m}$ is the average heat flux, $L$ is the wall surface circumference, $k$ is the thermal conductivity, and $\bar{w}$ is the dimensionless average flow velocity.

L-shaped region: the governing equation of the left rectangle is expressed as follows:

Momentum equation:

$$
\frac{\partial^{2} W_{1}}{\partial X^{2}}+\frac{\partial^{2} W_{1}}{\partial Y^{2}}=-\frac{1}{\mu} \frac{\mathrm{d} P}{\mathrm{~d} Z},
$$

where $W_{1}$ is the velocity of microfluid in the left rectangle.

Boundary conditions:

$$
\begin{aligned}
\frac{\partial W_{1}}{\partial Y}(X, 0) & =0, \\
\frac{\partial W_{1}}{\partial X}(0, Y) & =0, \\
W_{1}\left(X, \frac{a H}{2}\right) & =\beta_{w} \lambda \frac{\partial W_{1}}{\partial y}\left(X, \frac{a H}{2}\right),
\end{aligned}
$$

where $\beta_{w} \lambda$ is the velocity slip coefficient, $\beta_{w}=(2-F / F), F$ is the tangential momentum accommodation coefficient, and the Knudsen number is $k n=\lambda / a$.

For the L-shaped region, the governing equation of the right rectangle is expressed as follows:

Momentum equation:

$$
\frac{\partial^{2} W_{2}}{\partial X^{2}}+\frac{\partial^{2} W_{2}}{\partial Y^{2}}=-\frac{1}{\mu} \frac{\mathrm{d} P}{\mathrm{~d} Z},
$$

where $W_{2}$ is the velocity of microfluid in right rectangle.

Boundary conditions:

$$
\begin{aligned}
\frac{\partial W_{2}}{\partial Y}(X, 0) & =0, \\
\frac{\partial W_{2}}{\partial Y}(1, Y) & =0, \\
W_{2}\left(X, \frac{a-b}{2} H\right) & =\beta_{w} \lambda \frac{\partial W_{2}}{\partial Y}\left(X, \frac{(a-b) H}{2}\right) .
\end{aligned}
$$

Moreover, at the juncture of two rectangles, the two regions have equal velocity and shear force, and the following boundary conditions are obtained: 


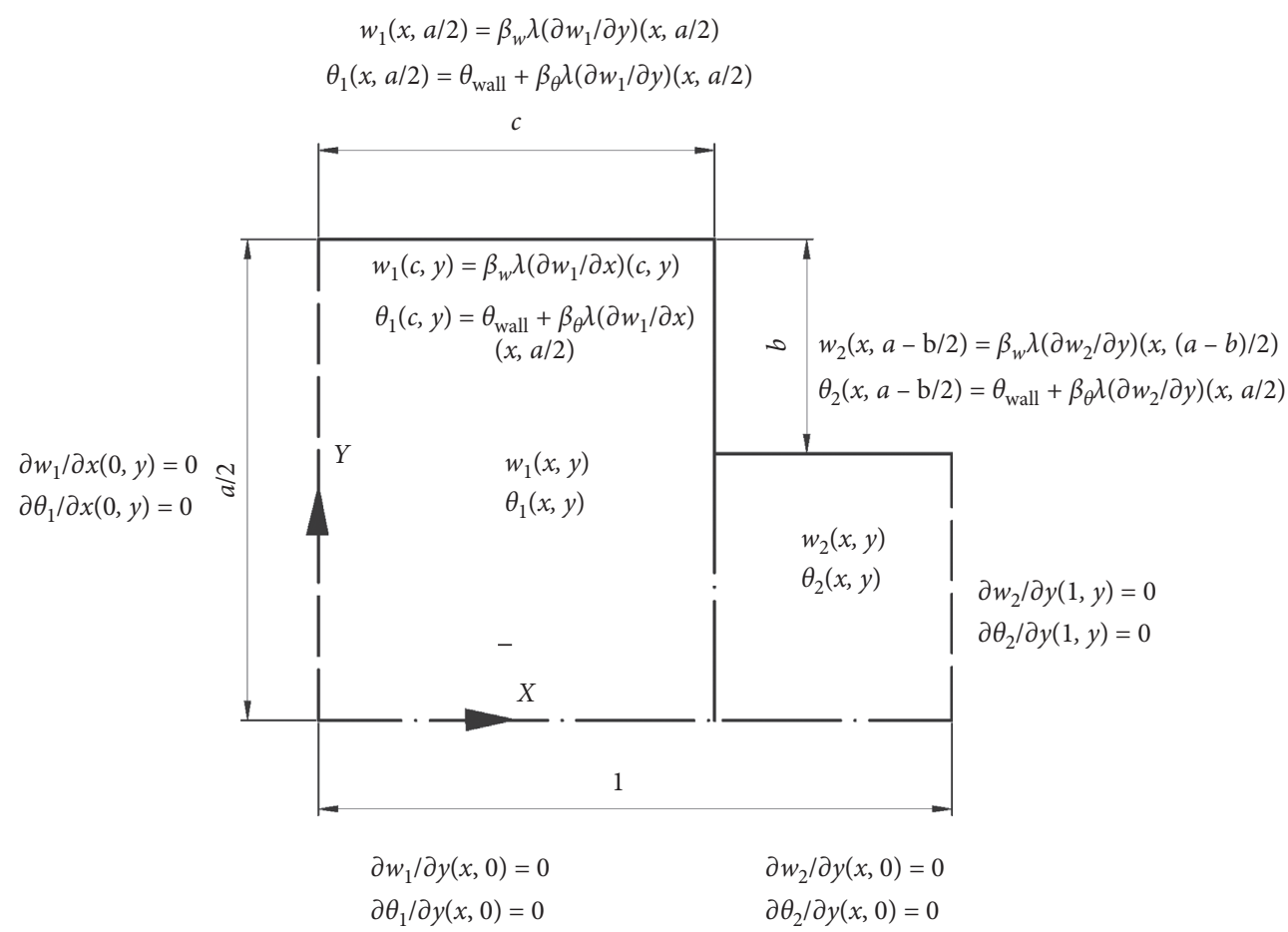

Figure 3: L-shaped region and boundary conditions.

$$
\begin{array}{cc}
W_{1}(c, Y)=W_{2}(c, Y), & 0 \leq Y<\frac{a H}{2-b H}, \\
\frac{\partial W_{1}(c, Y)}{\partial X}=\frac{\partial W_{2}(c, Y)}{\partial X}, & 0 \leq Y<\frac{a H}{2-b H} .
\end{array}
$$

The velocity dimensionless parameters are defined as $w=-W /\left(H^{2} \mathrm{~d} p / \mu \mathrm{d} Z\right), \quad x=X / H, \quad$ and $y=Y / H$. The microchannel governing equation is nondimensionalized and expressed as follows.

The dimensionless momentum equation of Region 1 is expressed as follows:

$$
\frac{\partial^{2} w_{1}}{\partial x^{2}}+\frac{\partial^{2} w_{1}}{\partial y^{2}}=-1,
$$

where $w_{1}$ is the dimensionless velocity of the microfluid in the left rectangle.

Boundary conditions:

$$
\begin{aligned}
\frac{\partial w_{1}}{\partial y}(x, 0) & =0 \\
\frac{\partial w_{1}}{\partial x}(0, y) & =0 \\
w_{1}\left(x, \frac{a}{2}\right) & =a \beta_{w} K n \frac{\partial w_{1}}{\partial y}\left(x, \frac{a}{2}\right) .
\end{aligned}
$$

The dimensionless momentum equation of Region 2 is expressed as follows:

$$
\frac{\partial^{2} w_{2}}{\partial x^{2}}+\frac{\partial^{2} w_{2}}{\partial y^{2}}=-1,
$$

where $W_{2}$ is the dimensionless velocity of the microfluid in the right rectangle.

Boundary conditions:

$$
\begin{aligned}
\frac{\partial w_{2}}{\partial y}(x, 0) & =0 \\
\frac{\partial w_{2}}{\partial y}(1, y) & =0 \\
w_{2}\left(x, \frac{a-b}{2}\right) & =a \beta_{w} K n \frac{\partial w_{2}}{\partial Y}\left(x, \frac{(a-b)}{2}\right) .
\end{aligned}
$$

The analytic solution of dimensionless velocity distribution of the aforesaid two regions is expressed as follows:

$$
\begin{aligned}
w_{1}(x, y)= & \frac{a^{2}}{8}-\frac{a^{2} \beta_{w} K n}{2}-\frac{y^{2}}{2} \\
& +\sum_{n} A_{n} \cos \left(\alpha_{n} y\right)\left(e^{\alpha_{n}(x-c)}+e^{-\alpha_{n}(x+c)}\right) .
\end{aligned}
$$

The eigenvalue $\alpha_{n}$ can be expressed as follows:

$$
\begin{aligned}
1+ & a \beta_{w} K n \alpha_{n} \tan \frac{\alpha_{n} a}{2}=0 \\
w_{2}(x, y)= & \frac{1}{2}\left(\frac{a}{2}-b\right)^{2}-\frac{a^{2} \beta_{w} K n}{2}-\frac{y^{2}}{2} \\
& +\sum_{m} B_{m} \cos \left(\eta_{m} y\right)\left(e^{\eta_{m}(x-2+c)}+e^{-\eta_{m}(x-c)}\right) .
\end{aligned}
$$


The eigenvalue $\eta_{m}$ of the above equation can be expressed as follows:

$$
1+a \beta_{w} K n \tan \left(\frac{a}{2}-b\right) \eta_{m}=0 .
$$

According to the velocity slip boundary conditions of microgroove,

$$
w_{2}\left(x, \frac{a-b}{2}\right)=a \beta_{w} K n \frac{\partial w_{2}}{\partial x}\left(x, \frac{(a-b)}{2}\right) .
$$

The velocity of the left region can be expressed as follows:

$$
\begin{aligned}
& \sum_{n=1}^{N} A_{n} \cos \left(\alpha_{n} y_{i}\right)\left(1+e^{-2 \alpha_{n} c}\right) \\
& \quad-a \beta_{w} K n \sum_{n=1}^{N} A_{n} \alpha_{n} \cos \left(\alpha_{n} y_{i}\right)\left(1-e^{-2 \alpha_{n} c}\right)=0 .
\end{aligned}
$$

Next, the velocity and shear stress of the two regions in the L-shaped domain can be matched along the common boundary. The conditions are given as

$$
\begin{array}{cc}
w_{1}(c, y)=w_{2}(c, y), \quad 0 \leq y<\frac{a}{2-b} \\
\frac{\partial w_{1}(c, y)}{\partial x}=\frac{\partial w_{2}(c, y)}{\partial x}, \quad 0 \leq y<\frac{a}{2-b} .
\end{array}
$$

The above two equations are changed to

$$
\begin{aligned}
& \sum_{n=1}^{N} A_{n} \cos \left(\alpha_{n} y_{i}\right)\left(1+e^{-2 \alpha_{n} c}\right) \\
& \quad-\sum_{m=1}^{M} B_{m} \cos \left(\eta_{m} y_{i}\right)\left(1+e^{-2 \eta_{m}(1-c)}\right)=\frac{\left(b^{2}-a b\right)}{2} \\
& \sum_{n=1}^{N} A_{n} \alpha_{n} \cos \left(\alpha_{n} y_{i}\right)\left(1-e^{-2 \alpha_{n} c}\right) \\
& \quad+\sum_{m=1}^{M} B_{m} \eta_{m} \cos \left(\eta_{m} y_{i}\right)\left(1-e^{-2 \eta_{m}(1-c)}\right)=0
\end{aligned}
$$

where in equations (17), (19), and (20), we choose $N$ points along the boundary at $x=c$ and obtain

$$
y_{i}=\frac{(i-1) a}{(2 N)}, \quad i=1 \text { to } N \text {. }
$$

We truncate $A_{n}$ to $N$ terms and $B_{m}$ to $M$ terms. Note that $M$ can be calculated from the following equation:

$$
M=\text { floor function }\left[N\left(\frac{1-2 b}{a}\right)\right]+1 \text {, }
$$

where $N=40$

The linear system of the $M+N$ equations with $M+N$ unknowns is solved for the coefficients of $A_{n}$ and $B_{m}$.

Substituting $A_{n}$ and $B_{m}$ into the velocity profiles (equations (12) and (14)), the velocity of the microfluid flow can be obtained.

The mean value for $w(x, y)$ is derived as

$$
\bar{w}=\frac{1}{(a / 2)-b(1-c)} \int_{0}^{c} \int_{0}^{a / 2} w_{1} \mathrm{~d} y \mathrm{~d} x+\int_{c}^{1} \int_{0}^{a / 2-b} w_{2} \mathrm{~d} y \mathrm{~d} x .
$$

Furthermore, $f$ Re can be expressed as a function of the volumetric flow rate in the following equation:

$$
f \operatorname{Re}=\frac{D_{h}^{2}}{2 w_{\text {mean }} H^{2}}
$$

where $D_{h}$ is the hydraulic diameter. In a more detailed form,

$$
f \operatorname{Re}=\frac{2[a-2 b(1-c)]^{2}}{(1+b)^{2} \bar{w}}
$$

The velocity distribution can be obtained by the above calculations; however, the temperature and heat transfer data are also important parts of a microchannel fluid. The calculation of the temperature distribution is described below.

And, the present paper is the temperature jump problem describing highly conductive boundaries. The temperature is temperature jump conditions on the boundary for each cross section and varies linearly downstream. The given heat flux varies peripherally but is constant with respect to axial distance.

First of all, the calculated analytic solution of velocity distribution is substituted into the temperature distribution function to obtain the governing equation for the dimensionless temperature distribution of fluid in the microchannel:

$$
\begin{gathered}
\frac{\partial^{2} \theta_{1}(x, y)}{\partial x^{2}}+\frac{\partial^{2} \theta_{1}(x, y)}{\partial y^{2}}=\frac{a^{2}}{8}-\frac{a^{2} \beta_{w} K n}{2}-\frac{y^{2}}{2} \\
+\sum_{n} A_{n} \cos \left(\alpha_{n} y\right)\left(e^{\alpha_{n}(x-c)}+e^{-\alpha_{n}(x+c)}\right) .
\end{gathered}
$$

The eigenvalue $\alpha_{n}$ is expressed as

$$
1+a \beta_{w} K n \alpha_{n} \tan \frac{\alpha_{n} a}{2}=0 .
$$

The boundary conditions can be expressed as 


$$
\begin{aligned}
\frac{\partial \theta_{1}}{\partial y}(x, 0) & =0, \\
\frac{\partial \theta_{1}}{\partial x}(0, y) & =0, \\
\theta_{1}\left(x, \frac{a}{2}\right) & =\theta_{\text {wall }}+a \beta_{w} K n \beta \frac{\partial w_{1}}{\partial y}\left(x, \frac{a}{2}\right), \\
\theta_{1}(c, y) & =\theta_{\text {wall }}+a \beta_{w} K n \beta \frac{\partial w_{1}}{\partial x}\left(x, \frac{a}{2}\right), \\
\frac{\partial \theta_{2}(x, y)}{\partial x^{2}} & +\frac{\partial \theta_{2}(x, y)}{\partial y^{2}}=\frac{1}{2}\left(\frac{a}{2}-b\right)^{2}-\frac{a^{2} \beta_{w} K n \beta}{2}-\frac{y^{2}}{2} \\
& +\sum_{m} B_{m} \cos \left(\eta_{m} y\right)\left(e^{\eta_{m}(x-2+c)}+e^{-\eta_{m}(x-c)}\right),
\end{aligned}
$$

where $\theta_{1}$ and $\theta_{2}$ are local dimensionless temperatures and $\beta=\beta_{\theta} / \beta_{w}$.

Eigenvalue $\eta_{m}$ :

$$
1+a \beta_{w} K n \tan \left(\frac{a}{2}-b\right) \eta_{m}=0 .
$$

The boundary conditions can be expressed as

$$
\begin{aligned}
\frac{\partial \theta_{2}}{\partial y}(x, 0) & =0, \\
\frac{\partial \theta_{2}}{\partial y}(1, y) & =0, \\
\theta_{2}\left(x, \frac{a-b}{2}\right) & =\theta_{\text {wall }}+a \beta_{w} K n \beta \frac{\partial w_{2}}{\partial y}\left(x, \frac{a}{2}\right),
\end{aligned}
$$

where $\theta_{w}$ is set as 0 , and the temperature boundary condition is substituted into the aforesaid temperature distribution governing equation. The temperature distribution function can thus be worked out:

$$
\begin{aligned}
\theta_{1}(x, y)= & \frac{a^{2}}{384}-\frac{a^{4}}{64}-\frac{a^{4}}{4} a^{2} \beta_{w}^{2} K n^{2} \beta^{2} \\
& +\left(\frac{a^{2}}{8}-\frac{a^{2}}{2} \beta_{w} K n \beta\right) \frac{y^{2}}{2}-\frac{y^{4}}{24}+ \\
\sum_{n} \frac{C_{n}}{2 \alpha_{n}} x \cos \left(\alpha_{n} y\right)\left[e^{\alpha_{n}(x-c)}+e^{-\alpha_{n}(x+c)}\right] & \\
& +\sum_{n} D_{n} \cos \left(\alpha_{n} y\right)\left[e^{\alpha_{n}(x-c)}+e^{-\alpha_{n}(x+c)}\right] \\
\theta_{2}(x, y)= & \frac{4}{25}\left(\frac{a}{2}-b\right)^{4}-\frac{\beta \lambda_{\theta} a}{8}\left(\frac{a}{2}-b\right)^{2}-\frac{\beta \lambda_{\theta}}{2}\left(\frac{a}{2}-b\right)^{2} \\
& -\frac{\beta^{2} \lambda_{\theta}^{2}}{4} a\left(\frac{a}{2}-b\right)-\frac{\beta \lambda_{\theta}}{6}\left(\frac{a}{2}-b\right)^{3} \\
& +\sum_{m} \frac{E_{m}}{2 \eta_{m}} x \cos \left(\eta_{m} y\right)\left[e^{\eta_{n}(x-2+c)}-e^{-\eta_{m}(x-c)}\right] \\
& +\sum_{m} F_{m} \cos \left(\eta_{m} y\right)\left[e^{\eta_{n}(x-2+c)}-e^{-\eta_{m}(x-c)}\right] .
\end{aligned}
$$

Temperature distribution equations (31) and (32) and coefficients $C_{n}, D_{n}, E_{m}$, and $F_{m}$ are calculated using the modified point matching method. The boundary conditions are expressed as follows:

$$
\begin{aligned}
& \theta_{1}(c, y)=\theta_{\text {wall }}+a \beta_{w} K n \beta \frac{\partial w_{1}}{\partial x}\left(x, \frac{a}{2}\right), \\
& \theta_{1}(c, y)=\frac{b+c}{1-c} \theta_{2}(c, y), \quad 0 \leq y<\frac{a}{2-b}, \\
& \frac{\partial \theta_{1}(c, y)}{\partial x}=\frac{b+c}{1-c} \frac{\partial \theta_{2}(c, y)}{\partial x}, \quad 0 \leq y<\frac{a}{2-b} .
\end{aligned}
$$

Equations (30)-(32) are expanded to obtain the following three equations: 


$$
\begin{aligned}
& \sum_{n=1}^{2 N} \frac{C_{n}}{2 \alpha_{n}} \cos \left(\alpha_{n} y_{i}\right)\left\{\left(1+e^{-2 \alpha_{n}} c\right)-a \beta_{w} K n \beta\left[1+e^{-2 \alpha_{n} c}+c \alpha_{n}\left(1-e^{-2 \alpha_{n} c}\right)\right]\right\} \\
& +\sum_{n=1}^{2 N} D_{n} \cos \left(\alpha_{n} y_{i}\right)\left[\left(1+e^{-2 \alpha_{n} c}\right)-a \beta_{w} K n \beta \alpha_{n}\left(1-e^{-2 \alpha_{n} c}\right)\right] \\
& =\frac{y_{i}^{4}}{24}-\left(\frac{a^{2}}{8}-\frac{a^{2} \beta_{w} K n \beta}{2}\right) \frac{y^{2}}{2}+\frac{a^{4} \beta_{w}^{4} K n^{2} \beta^{2}}{4}+\frac{a^{4}}{64}-\frac{a^{2}}{384}, \quad i=2 M+1 \text { to } 2 N, \\
& \sum_{n=1}^{2 N} \frac{C_{n}}{2 \alpha_{n}} x \cos \left(\alpha_{n} y_{i}\right)\left[1+e^{-2 \alpha_{n} c}\right]+\sum_{n=1}^{2 N} D_{n} \cos \left(\alpha_{n} y_{i}\right)\left[1+e^{-2 \alpha_{n} c}\right]-\frac{b+c}{1-c} \sum_{m=1}^{2 M} \frac{E_{m}}{2 \eta_{m}} x \cos \left(\eta_{m} y_{i}\right)\left[e^{\eta_{n}(2 c-2)}-1\right] \\
& -\frac{b+c}{1-c} \sum_{m=1}^{2 M} F_{m} \cos \left(\eta_{m} y_{i}\right)\left[e^{\eta_{n}(2 c-2)}-1\right] \\
& =\frac{4}{25}\left(\frac{a}{2}\right)^{4}-\frac{a \beta_{w} K n \beta}{2}\left(\frac{a}{2}-b\right)^{2}\left(\frac{a}{4}+1\right)-\left[\frac{a^{3} \beta_{w}^{2} K n^{2} \beta^{2}}{4}\left(\frac{a}{2}-b\right)-a\right] \\
& -\frac{a \beta_{w} K n \beta}{6}\left(\frac{a}{2}-b\right)^{3}+\frac{y_{i}^{2}}{4}\left[\left(\frac{a}{2}-b\right)^{2}-a^{2} \beta_{w} K n \beta-2\left(a^{2}-\frac{a^{2} \beta_{w} K n \beta}{2}\right)\right]-\frac{a^{2}}{384}+\frac{a^{2}}{64}, \quad i=1 \text { to } 2 M \text {, } \\
& \sum_{n=1}^{2 N} \frac{C_{n}}{2 \alpha_{n}} x \cos \left(\alpha_{n} y_{i}\right)\left[\left(1+e^{-2 \alpha_{n} c}\right)+c \alpha_{n}\left(1-e^{-2 \alpha_{n} c}\right)\right]+\sum_{n=1}^{2 N} D_{n} \cos \left(\alpha_{n} y_{i}\right)\left[\alpha_{n}\left(1+e^{-2 \alpha_{n} c}\right)\right] \\
& -\frac{b+c}{1-c} \sum_{m=1}^{2 M} \frac{E_{m}}{2 \eta_{m}} x \cos \left(\eta_{m} y_{i}\right)\left[\left(e^{\eta_{n}(2 c-2)}-1\right)+\frac{c(b+c)}{1-c} \eta_{m}\left(e^{\eta_{n}(2 c-2)}+1\right)\right] \\
& +\sum_{m=1}^{2 M} F_{m} \cos \left(\eta_{m} y_{i}\right)\left[e^{\eta_{n}(2 c-2)}+1\right]=0, \quad i=1 \text { to } 2 M
\end{aligned}
$$

where $y_{i}$ in equations (34)-(36), we choose $2 N$ points along the boundary at $x=c$ and obtain

$$
y_{i}=\frac{(i-1) a}{(2 N)}, \quad i=1 \text { to } 2 N .
$$

And, truncate $C_{n}$ and $D_{n}$ to $2 N$ terms and $E_{m}$ and $F_{m}$ to $2 M$ terms. Note that $M$ can be calculated from the following equation:

$$
M=\text { floor function }\left[2 N\left(1-2 \frac{b}{a}\right)\right]+1 .
$$

The linear system of $2 M+2 N$ equations with $2 M+2 N$ unknowns is solved for the coefficients of $C_{n}, D_{n}, E_{m}$, and $F_{m}$.

The numerical calculations in the present study were based on a new numerical analysis scheme that was used to name modified point-matching methods. In order to estimate the series convergence, the present research tests the term of the coefficients $N$. When the term of the coefficient $N$ is set as $N=40$, the precision of the coefficient convergences to four decimal places. Therefore, the present investigation uses $N=60$ to calculate the results in order to ensure convergence.

Moreover, the heat flux in the microchannel with microgrooves is calculated as follows:

$$
\begin{aligned}
\bar{q} \equiv & \left.\frac{1}{1+(a / 2)-b} \int_{0}^{1-c} \frac{\partial \theta_{1}}{\partial y}\right|_{y=a / 2} \mathrm{~d} x \\
& +\left.\left.\int_{(a / 2)-b}^{a / 2} \frac{\partial \theta_{1}}{\partial x}\right|_{x=c} \mathrm{~d} y \int_{1-c}^{1} \frac{\partial \theta_{2}}{\partial y}\right|_{y=(a / 2)-b} .
\end{aligned}
$$

The mean Nusselt number is calculated as follows:

$$
N u \equiv \frac{\bar{q}}{\text { Area } \cdot\left(T_{\text {wall }}-T_{\text {mean }}\right)} .
$$

\section{Results and Discussion}

In the first section of the results and discussion, for validating the convergence of the series, the present study predicts the term of the coefficients. When the term of series $N$ is set as $N=40$, the precision of the coefficient convergences to five decimal places. Therefore, the present investigation will apply $N=60$ to compute the numerical data from Figures 4-8 for ensuring convergence. Table 1 shows some selected coefficients obtained by the modified pointmatching method under $N=40$. The numerical solution in the present investigation can be exactly computed by the modified point-match methods. Convergence is fairly fast. For realizing coefficients $A_{n}, B_{m}, C_{n}, D_{n}, E_{m}$, and $F_{m}$, the values of the some chosen coefficients are listed in Table 1. 

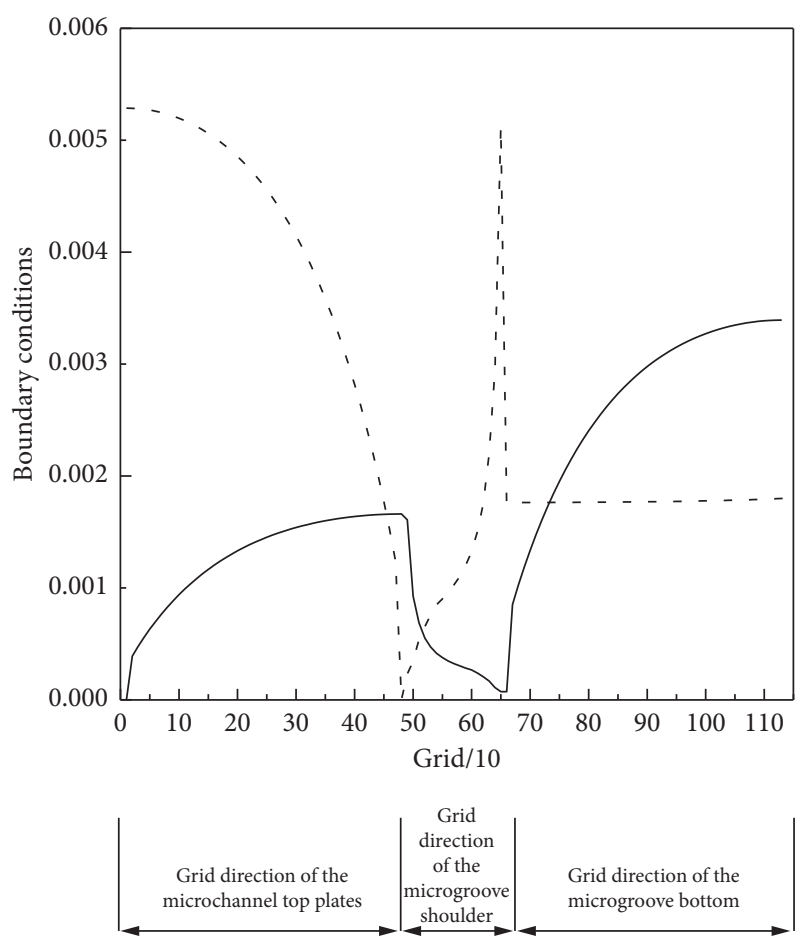

- - - Velocity slip boundary conditions

— Temperature jump boundary conditions

FIGURE 4: Slip velocity and discontinuous temperature boundary conditions along the microchannel top, microgroove shoulder and microgroove bottom $\left(\beta_{w} K n=0.02\right)$.

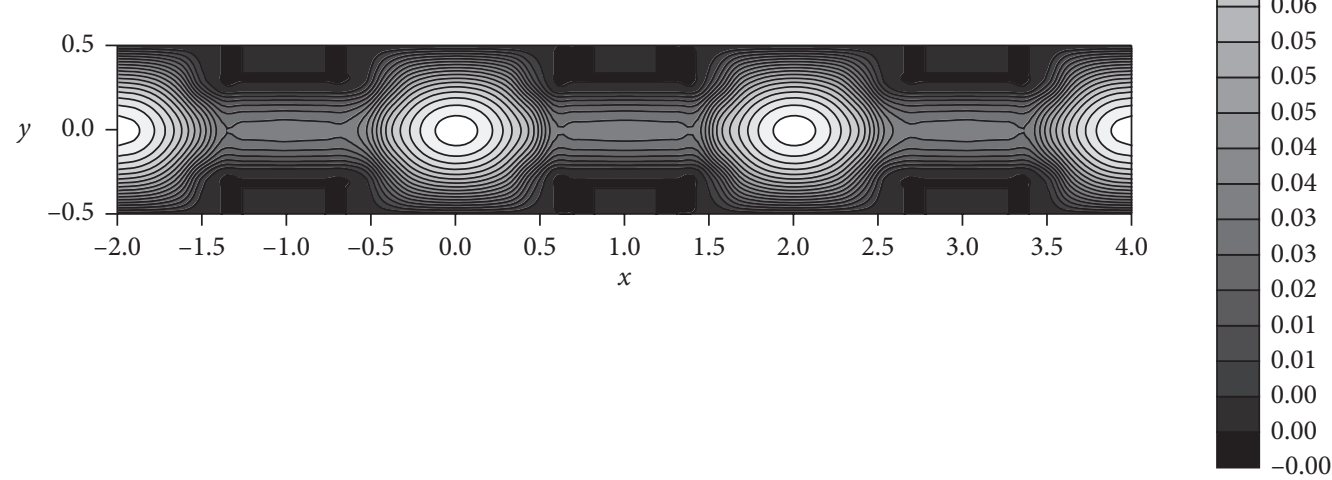

FIgURE 5: Local velocity distribution of the microchannel and microgroove $\left(\beta_{w} K n=0.025\right)$.

Figure 9 shows the applied grids in the present investigation. Figure 10 discusses the velocity vector of the microchannel with microgrooves. Figure 10 represents velocity vector of the L-shaped region based on the contour map of the fluid velocity. The present investigation is based on the two-dimensional analysis of microchannel. The main flow direction of the velocity in the microchannel with microgrooves was normal in this paper ( $z$-dimension). It is observed that the velocity vectors in microchannel with microgrooves decrease from the center of the domain $(x=0$, $0 \leq y \leq(a / 2) ; y=0,0 \leq x \leq 1)$ to the boundary of the domain $(y=(a / 2), \quad 0 \leq x \leq c ; x=c,(a / 2)-b \leq y \leq(a / 2) ; y=(a / 2)-b$, $c \leq x \leq 1)$.

Figure 4 presents the boundary slip velocity and discontinuous temperature distribution trend of microchannel and microgroove. The slip velocities and discontinuous temperatures at the top of the microchannel, as well as the shoulder and bottom of the microgroove, are shown in 


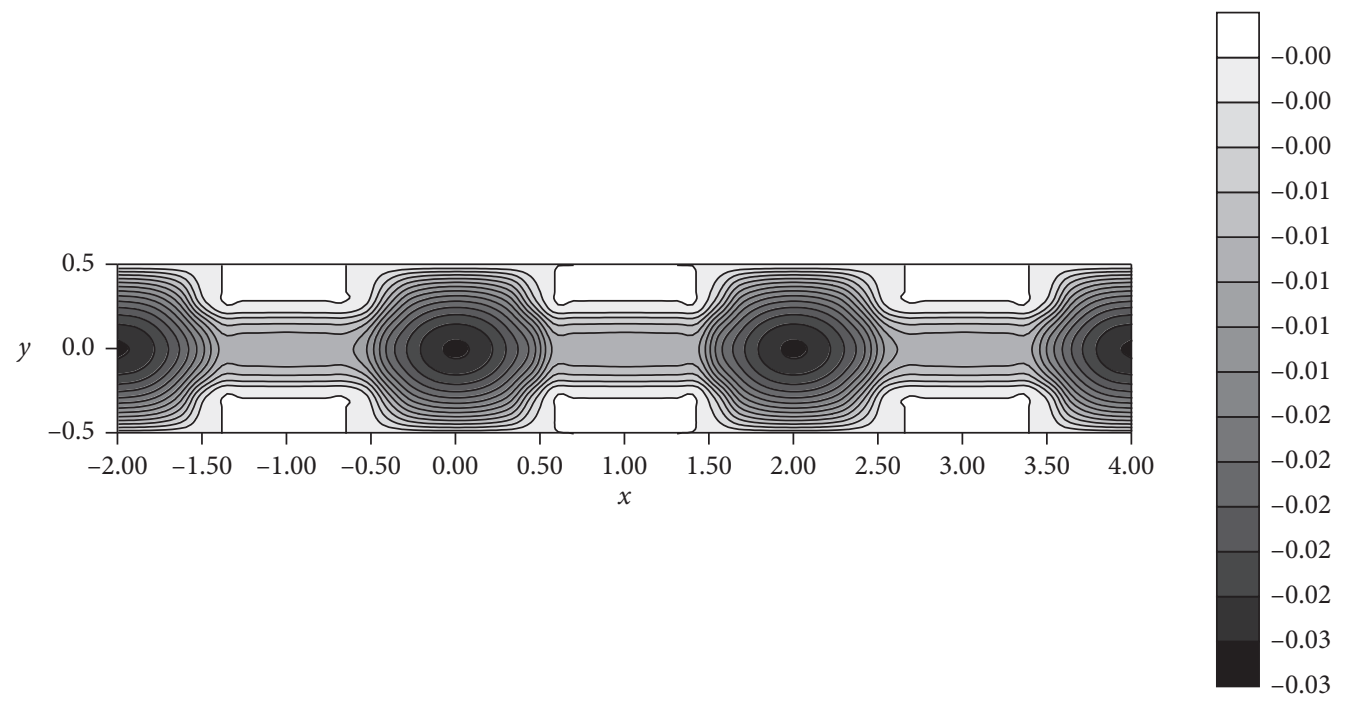

Figure 6: Local temperature distribution of the microchannel and microgroove $\left(\beta_{w} K n=0.02\right)$.

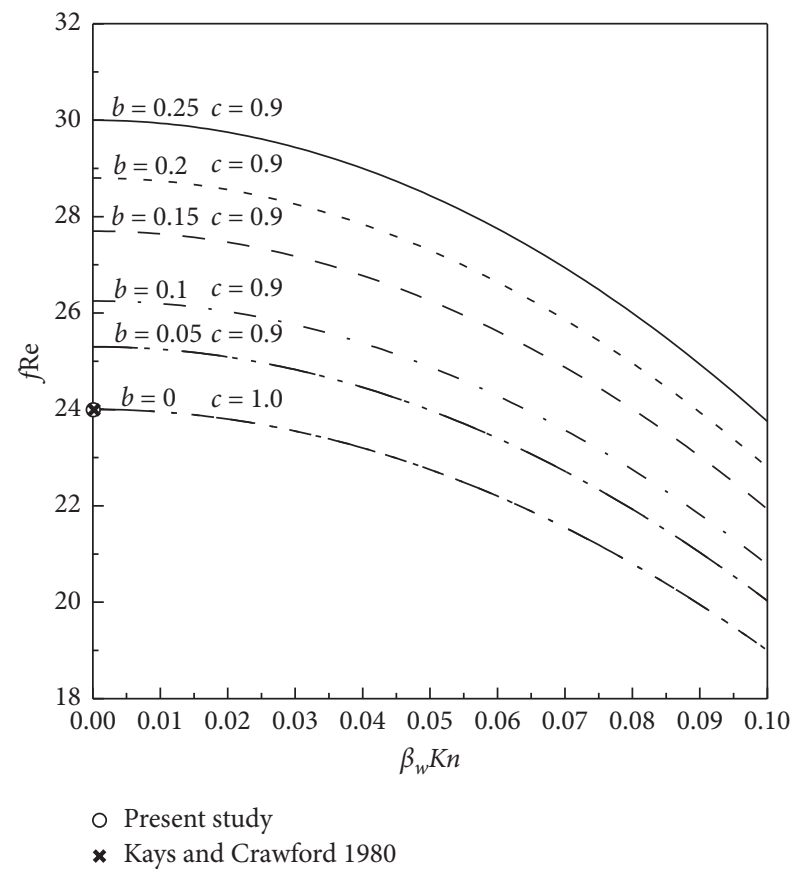

Figure 7: Effect of $\beta_{w} K n$ on the friction factor.

Figure 4. It was observed that the slip velocity decreased gradually at the top of the microchannel under the shear force of the microgroove shoulder, whereas the slip velocity increased gradually at the shoulder of the microgroove far from the top of microchannel and the variability decreased at the bottom of microgroove. The discontinuous temperature rose at the top of the microchannel under the thermal effect of the microgroove shoulder, dropped at the shoulder of the microgroove far from the top of the microchannel, and rose at the bottom of the microgroove.

Figures 5 and 6 show the local velocity distribution of the microchannel and microgroove and the local temperature distribution of the microchannel and microgroove, respectively $\left(\beta_{w} K n=0.025\right)$. It was observed that the local

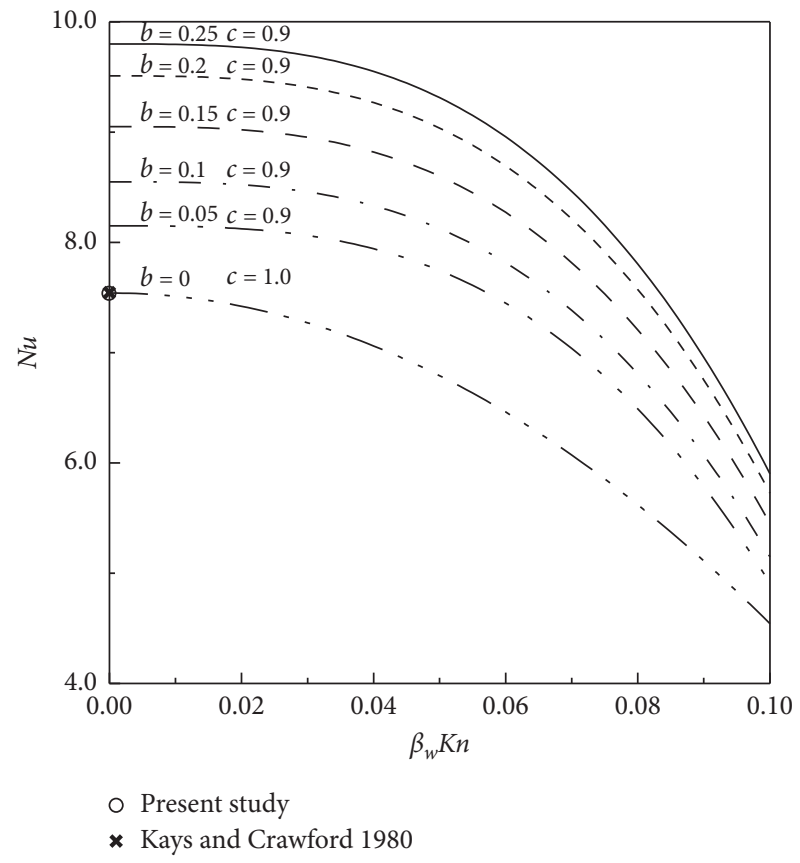

Figure 8: Effect of $\beta_{w} K n$ on $\mathrm{Nu}$.

velocity was lower at the slip boundary and that the local velocity increased as the distance to the geometric center shortened. The local temperature distribution presented a larger value on the wall surface. This study assumed there was a linear temperature change in the $z$ direction and that the temperature of each section in the $z$ direction was constant. It was observed that the temperature dropped as the distance to the center shortened; on the contrary, the closer to the wall surface of microchannel and grooves, the higher the temperature became.

The node-density effect on the predicted solutions, in terms of $f \mathrm{Re}$ and $N u$, is calculated under different grid size and is listed in Table 2. The parameters setting in Table 2 is under $\beta_{w} K n=0, c=1.0$, and $b=0$. That is equal to the 
TABLE 1: Selected coefficients for estimating the precision of the coefficients.

\begin{tabular}{lccccccc}
\hline \multicolumn{7}{c}{$a=1, b=0.25, c=0.5, \beta_{w} K n=0.02, N=40$} \\
\hline$A_{1}=-0.47562$ & $A_{2}=-0.00017$ & $A_{3}=0.01003$ & $A_{5}=-0.00237$ & $A_{10}=0.00002$ & $A_{20}=0.00001$ & $A_{39}=-0.00000$ & $A_{40}=0.00000$ \\
$B_{1}=0.00401$ & $B_{2}=-0.00001$ & $B_{3}=0.00135$ & $B_{5}=0.00077$ & $B_{10}=-0.00019$ & $B_{15}=0.00007$ & $B_{19}=-0.00000$ & $B_{20}=0.00000$ \\
$C_{1}=-0.23165$ & $C_{2}=0.18751$ & $C_{3}=-0.11679$ & $C_{10}=0.054136$ & $C_{40}=0.00621$ & $C_{60}=0.00000$ & $C_{79}=0.00000$ & $C_{80}=-0.00000$ \\
$D_{1}=-0.19657$ & $D_{2}=0.14532$ & $D_{3}=-0.09573$ & $D_{10}=0.03472$ & $D_{40}=-0.00411$ & $D_{60}=0.00000$ & $D_{79}=0.00000$ & $D_{80}=-0.00000$ \\
$E_{1}=0.09673$ & $E_{2}=-0.07462$ & $E_{3}=-0.05675$ & $E_{10}=0.00034$ & $E_{20}=-0.00013$ & $E_{30}=0.00002$ & $E_{39}=0.00000$ & $E_{40}=0.00000$ \\
$F_{1}=0.012473$ & $F_{2}=-0.10435$ & $F_{3}=-0.08726$ & $F_{10}=0.00081$ & $F_{20}=-0.00036$ & $F_{30}=0.00006$ & $F_{39}=0.00000$ & $F_{40}=-0.00000$ \\
\hline
\end{tabular}

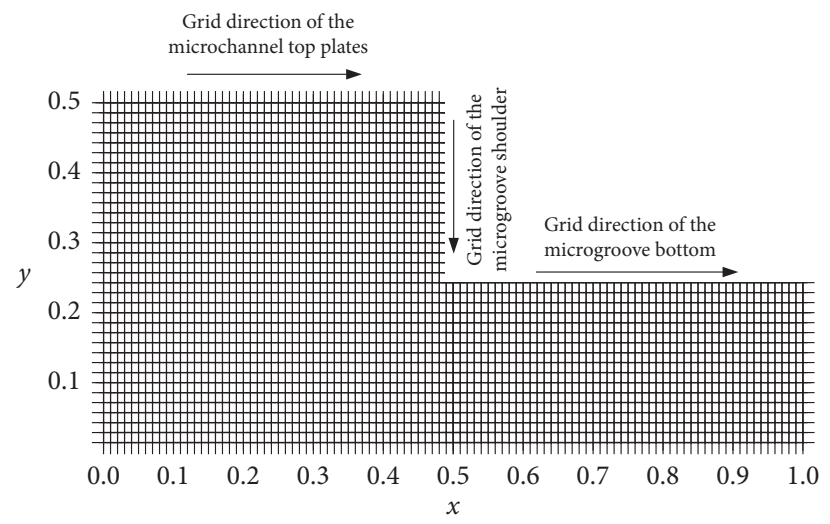

FIGURE 9: Schematic diagram of the grids and schematic directions of the boundaries.

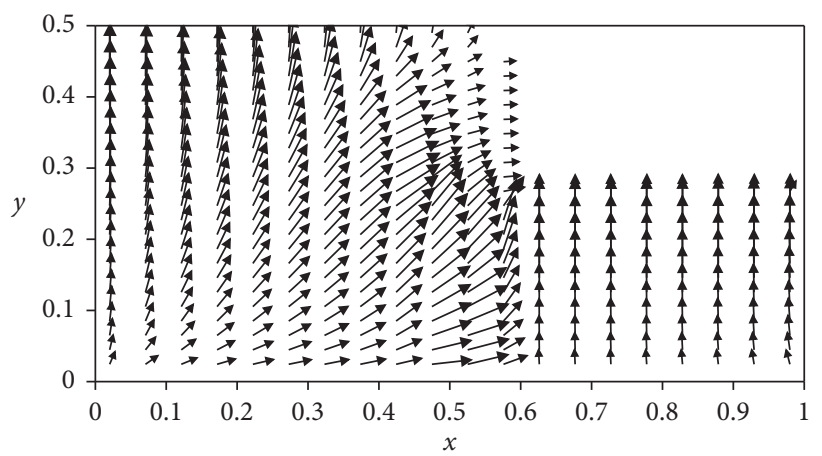

FIGURE 10: Velocity vector of L-shape region.

TABLe 2: The test results of grid independency for $f \mathrm{Re}$ and $N u$ and computational time.

\begin{tabular}{|c|c|c|c|c|c|c|c|}
\hline \multicolumn{8}{|c|}{ Analytical solution $f \mathrm{Re}=24, \mathrm{Nu}=7.54$} \\
\hline Grids & $f \operatorname{Re}$ & Error & $\mathrm{Sec}$ & Grids & $N u$ & Error & Sec \\
\hline $20 \times 20$ & 21.165 & 11.8 & 0.3 & $40 \times 40$ & 6.826 & 9.45 & 4.7 \\
\hline $30 \times 30$ & 23.834 & 0.69 & 0.9 & $60 \times 60$ & 7.173 & 4.87 & 10.2 \\
\hline $40 \times 40$ & 23.926 & 0.31 & 2.3 & $80 \times 80$ & 7.531 & 0.12 & 16.7 \\
\hline $60 \times 60$ & 24.016 & 0.00 & 6.1 & $120 \times 120$ & 7.542 & 0.02 & 22.8 \\
\hline $80 \times 80$ & 24.016 & -0.00 & 12.6 & $160 \times 160$ & 7.542 & 0.02 & 30.4 \\
\hline
\end{tabular}

special case for the laminar flow between two infinitely long plates [21], and $f \operatorname{Re}=24$ and $N u=7.54$. The relative percent errors for $f \operatorname{Re}$ and $\mathrm{Nu}$ are defined as

$$
\text { error }=\frac{\text { analytical solution }- \text { present results }}{\text { analytical solution }} \times 100 \% \text {. }
$$

For the density of $40 \times 40$ grid size, the relative error of $f \operatorname{Re}$ values is less than $0.31 \%$, and for the density of $80 \times 80$ grid size, the relative error of $N u$ numbers is less than $0.12 \%$. Besides, the hardware of the computer applying in the present numerical analysis uses 4-core 3.4 GHZ CPU and 8G DDR3-1600 RAM. The computational time, seconds, are also listed in Table 2.

Figure 7 shows the effect of rarefaction parameter $\beta_{w} K n$ on the $f \mathrm{Re}$ under different dimensionless microgroove depths. First, for validating the fluid dynamic characteristic of the present study, one set the present parameters of 
microchannel with the microgrooves as $c=1.0, b=0$, and $\beta_{w} K n=0$. That is equal to the special case for the laminar flow between two infinitely long plates [21], and $f R e=24$. Under the special case, according to the calculated results of the present numerical methods, $f$ Re value under the special case is exactly 24.017. Figure 7 shows that the results of the present paper are in a good agreement with the velocity characteristic of laminar flow between two infinitely long plates.

Moreover, dimensionless depth $b$ of the microgroove was increased. As the contact area of the fluid and the shoulder wall surface was enlarged, the $f$ Re increased. It was observed that the fRe decreased as rarefaction parameter $\beta_{w} K n$ increased, because the fluid was rarefied and the friction was reduced when the rarefaction effect was enhanced.

Figure 8 shows the effect of rarefaction parameter $\beta_{w} K n$ on $\mathrm{Nu}$ under different dimensionless microgroove lengths. Next, for further validating the thermocharacteristic of the present investigation, the conditions of the present microchannel with microgrooves is set the same as the laminar fluid flow between two infinitely long plates [21], i.e., under $\beta_{w} K n=0, c=1.0$, and $b=0$, the Nusselt number was similar to the laminar flow between two infinitely long plates. Based on the numerical analysis of the present modified pointmatching method, the Nusselt number under the special case is 7.542, i.e., under the present theoretical method prediction, the Nusselt number approximated the $N u$ of the laminar fluid flow between two infinitely long plates; the $\mathrm{Nu}$ equally is equal to 7.54. The data of the present study are in a good agreement with the thermocharacteristic of laminar flow between two infinitely long plates. The comparison is shown in Figure 8.

In addition, the average $N u$ decreased as rarefaction parameter $\beta_{w} K n$ increased. If dimensionless depth $b$ of the microgroove was increased, the heat transfer area would be enlarged and $\mathrm{Nu}$ would increase accordingly.

\section{Conclusion}

This study found that the slip velocity decreased gradually at the top of the microchannel under the shear force of the microgroove shoulder, whereas the slip velocity increased gradually at the shoulder of the microgroove far from the top of the microchannel. The variability decreased at the bottom of the microgroove. The discontinuous temperature rose at the top of the microchannel under the thermal effect of the microgroove shoulder, dropped at the shoulder of the microgroove far from the top of the microchannel, and rose at the bottom of the microgroove. Moreover, the contact area of the fluid and wall surface was enlarged when dimensionless depth $b$ of the microgroove was increased, the $f \mathrm{Re}$ increased accordingly, the fluid was rarefied as $\beta_{w} K n$ increased, and the $f$ Re was reduced. The fluid would be rarefied if $\beta_{w} K n$ was increased, and the average $N u$ would be reduced as a result. If dimensionless depth $b$ of the microgroove was increased, the average $N u$ would be increased as the heat transfer area was enlarged.

\section{Abbreviations}

a: Dimensionless half height of microchannel

$A_{n}$ : Coefficient of the function (equation (12))

$b$ : Dimensionless half height of the microgroove

$B_{m}$ : Coefficient of the function (equation (14))

$c$ : Dimensionless half spacing between width of the microchannel

$C_{n}: \quad$ Coefficient of the function (equation (31))

$D_{n}$ : Coefficient of the function (equation (31))

$D_{h}: \quad$ Hydraulic diameter $=(4[a-b(1-c)] H / 2+b-c)$ (m)

$E_{m}: \quad$ Coefficient of the function (equation (32))

$F_{m}: \quad$ Coefficient of the function (equation (32))

$F_{w}: \quad$ Tangential momentum accommodation coefficient

$F_{\theta}: \quad$ Thermal accommodation coefficient

$f$ Re: Friction factor-Reynolds number product

$H$ : $\quad$ Half span of the selected domain (m)

$K n$ : Knudsen number

$\mathrm{Nu}$ : Nusselt number

$P: \quad$ Pressure $\left(\mathrm{N} / \mathrm{m}^{2}\right)$

Re: $\quad$ Reynolds number $=\left[W_{z} D_{h} / \nu\right]$

$W_{z}$ : Velocity in the $z$ direction $(\mathrm{m} / \mathrm{s})$

$w$ : Dimensionless velocity in the $z$ direction $=\left[-W_{z} /\left(H^{2} \mathrm{~d} p / \mu \mathrm{d} z\right)\right]$

$w_{\text {mean }}$ : Dimensionless mean velocity of the microflow

$x: \quad$ Dimensionless horizontal axis $=[X / H]$

$X: \quad$ Horizontal axis $(\mathrm{m})$

$y: \quad$ Dimensionless vertical axis $=[Y / H]$

$Y: \quad$ Vertical axis (m)

$z$ : $\quad$ Dimensionless axis normal to this paper

\section{Greek Letters}

$\mu: \quad$ Viscosity $\left(\mathrm{N}-\mathrm{s} / \mathrm{m}^{2}\right)$

$\theta_{1}$ : Dimensionless

temperature $=\left(T_{1}(X, Y)-T_{\text {wall }} /(b+c) H \bar{q}\right) k w_{\text {mean }}$

$\theta_{2}: \quad$ Dimensionless temperature $=\left(T_{2}(X, Y)-T_{\text {wall }} /(1-c) H \bar{q}\right) k w_{\text {mean }}$

$\theta_{\text {wall }}$ : Dimensionless boundary condition of the temperature, $\theta_{\text {wall }}=0$

$\beta$ : Thermal expansion coefficient, $=\beta_{\theta} / \beta_{w}$

$\beta_{w}$ : Dimensionless variables $\left(2-F_{w} / F_{w}\right)$

$\beta_{\theta}$ : Dimensionless variables $\left(2-F_{\theta} / F_{\theta}\right)(2 R / 1+R)(1 / \operatorname{Pr})$.

\section{Data Availability}

The data used to support the findings of the present investigation are not available because our research data come from computer simulation.

\section{Conflicts of Interest}

The author declares there are have no conflicts of interest.

\section{Acknowledgments}

The author gratefully acknowledges the support provided to this project by the Ministry of Science and Technology of 
Taiwan under Contract Numbers MOST 109-2221-E-019011 and MOST 108-2221-E-019-030.

\section{References}

[1] E. M. Sparrow and S. H. Lin, "Laminar heat transfer in tubes under slip-flow conditions," Journal of Heat Transfer, vol. 84, no. 4, pp. 363-369, 1962.

[2] R. M. Inman, Heat Transfer for Laminar Slip Flow of a Rarefied Gas in a Parallel Plate Channel or a Circular Tube with Uniform Wall Temperature, NASA TN, Washington, DC, USA, 1964.

[3] F. E. Larrode, C. Housiadas, and Y. Drossinos, "Slip-slow heat transfer in circular tubes," International Journal of Heat and Mass Transfer, vol. 43, no. 15, pp. 2669-2680, 2000.

[4] W. Elenbaas, "Heat dissipation of parallel plates by free convection," Physica, vol. 9, no. 1, pp. 1-28, 1942.

[5] B. Gebhart, Y. Jaluria, R. L. Mahajan, and B. Sammakia, Buoyancy-induced Flows and Transport, Taylor \& Francis, Oxfordshire, UK, 1988.

[6] W. Aung, "Fully developed laminar free convection between vertical plates heated asymmetrically," International Journal of Heat and Mass Transfer, vol. 15, no. 8, pp. 1577-1580, 1972.

[7] S. Yu and T. A. Ameel, "Slip-flow heat transfer in rectangular microchannels," International Journal of Heat and Mass Transfer, vol. 44, no. 22, pp. 4225-4234, 2001.

[8] H. P. Kavehpour, M. Faghri, and Y. Asako, "Effects of compressibility and rarefaction on gaseous flows in microchannels," Numerical Heat Transfer, Part A: Applications, vol. 32, no. 7, pp. 677-696, 1997.

[9] M. Vasudeviah and K. Balamurugan, "Heat transfer of rarefied gases in a corrugated microchannel," International Journal of Thermal Sciences, vol. 40, no. 5, pp. 454-468, 2001.

[10] G. Tunc and Y. Bayazitoglu, "Heat transfer in rectangular microchannels," International Journal of Heat and Mass Transfer, vol. 45, no. 4, pp. 765-773, 2002.

[11] L. Ghodoossi and N. E. Eğrican, "Prediction of heat transfer characteristics in rectangular microchannels for slip flow regime and $\mathrm{H} 1$ boundary condition," International Journal of Thermal Sciences, vol. 44, no. 6, pp. 513-520, 2005.

[12] X. Zhu and Q. Liao, "Heat transfer for laminar slip flow in a microchannel of arbitrary cross section with complex thermal boundary conditions," Applied Thermal Engineering, vol. 26, no. 11-12, pp. 1246-1256, 2006.

[13] M. Bahrami, M. M. Yovanovich, and J. R. Culham, "Pressure drop of fully-developed, laminar flow in microchannels of arbitrary cross-section," Journal of Fluids Engineering, vol. 128, no. 5, pp. 1036-1044, 2006.

[14] X. Zhang, T. Zhao, S. Wu, and F. Yao, "Experimental study on liquid flow and heat transfer in rough microchannels," Advances in Condensed Matter Physics, vol. 2019, Article ID 1974952, 9 pages, 2019.

[15] Y. Elguennouni, M. Hssikou, J. Baliti, and M. Alaoui, "Thermal lattice Boltzmann model for nonisothermal gas flow in a two-dimensional microchannel," Mathematical Problems in Engineering, vol. 2020, Article ID 8638494, 13 pages, 2020.

[16] M. M. Peiravi and J. Alinejad, "Hybrid conduction, convection and radiation heat transfer simulation in a channel with rectangular cylinder," Journal of Thermal Analysis and Calorimetry, vol. 140, no. 6, pp. 2733-2747, 2020.

[17] J. Alinejad and M. M. Peiravi, "Numerical analysis of secondary droplets characteristics due to drop impacting on 3D cylinders considering dynamic contact angle," Meccanica, vol. 55, no. 10, pp. 1975-2002, 2020.
[18] I. Asshaari, A. Jedi, and K. D. Pati, "A weibull distribution: flow and heat transfer of nanofluids containing carbon nanotubes with radiation and velocity slip effects," Mathematical Problems in Engineering, vol. 2020, Article ID 9417598, 9 pages, 2020.

[19] M. Yuan, R. Mohebbi, M. M. Rashidi, Z. Yang, and M. A. Sheremet, "Numerical study of MHD nanofluid natural convection in a baffled U-shaped enclosure," International Journal of Heat and Mass Transfer, vol. 130, pp. 123-134, 2019.

[20] R. Mohebbi and M. M. Rashidi, "Numerical simulation of natural convection heat transfer of a nanofluid in an L-shaped enclosure with a heating obstacle," Journal of the Taiwan Institute of Chemical Engineers, vol. 72, pp. 70-84, 2017.

[21] W. M. Kays and M. E. Crawford, Convection Heat and Mass Transfer, McGraw-Hill, New York, NY, USA, 1980. 